\title{
Actinomycosis: An unusual complication following appendicitis
}

\author{
THOMAS A. CASTILLENTI, DO
}

CHARLES R. CONKLIN, DO

We present a report of a case of retroperitoneal actinomycosis 3 years after appendectomy for a ruptured appendix. Actinomycosis is an unusual infectious disease that occasionally occurs after enteric perforation. The literature is reviewed, and the pathogenesis, diagnosis, and treatment are discussed.

Surgical procedures that are performed in the abdominal cavity in the face of gastrointestinal perforation result in soft-tissue contamination by endogenous flora. Anaerobic and gram-negative abscesses frequently resultfrom appendiceal and other colonic perforations. These polymicrobial contaminations seldom yield infections involving indolent, slowgrowing saprophytes. Discovery of a mass weeks to years following violation of the gastrointestinal tract should raise suspicion of abscesses involving these otherwise infrequent pathogens. If present and not considered, delayed healing and chronic infection with sinus tract formation may result. Actinomyces israelii is an organism that exemplifies this scenario. Infections involving this organism are infrequent. $A$ israelii has fastidious culture characteristics and an indolent growth pattern.

The first clinical description of actinomycosis has been attributed to von Langenbeck in $1845 .{ }^{1}$ In 1877 , Bollinger described the cervicofacial form of the disease, "Lumpy Jaw," in cattle. Harz, in 1878, named the organism Acti-

From the Department of General Surgery, Doctors Hospital of Stark County, Inc, Massillon, Ohio, where, at the time this paper was written, Dr Castillenti was resident in general surgery; Dr Conklin is a staff surgeon (general). Currently, Dr Castillenti is a fellow in peripheral vascular surgery at Youngstown Osteopathic Hospital, Youngstown, Ohio.

Reprint requests to Thomas A. Castillenti, DO, 8575 Bayberry Dr, Howland, OH 44448. nomyces bovis. In the same year, Israel described the first human infection with this organism. Israel then collected 37 similar cases in only 7 years. $^{2}$

The clinical spectrum of actinomycotic infections is customarily divided into three types: cervicofacial, abdominal, and thoracic. Cope's ${ }^{3}$ extensive review of 1300 cases showed cervicofacial involvement to predominate in more than half of these cases. One quarter of the cases were found to be abdominal, and 15\% were thoracic; few were localized to other sites.

The vast majority of cases of abdominal actinomycosis develop weeks, or even months, after enteric perforation. In most cases, involvement of intestinal mucosa is not demonstrated. Therefore, the terms gastrointestinal, ileocecal, appendiceal, and abdominal actinomycosis are, in fact, misnomers. Typically, retroperitoneal spread is found, usually in the musculature of the iliac fossa, because appendiceal rupture is the most common source of contamination. This fact was well demonstrated in the series of 122 cases of abdominal actinomycosis described by Putman and associates. ${ }^{4}$ In that series, 88 cases were attributed to appendiceal disease in one form or another; 62 cases were from ruptured appendixes. ${ }^{4}$ Acidity and rapid transit of the contents of the stomach and small bowel are believed to contribute to the low frequency of involvement in these areas.

Apart from the cecum, actinomycosis of the colon is a rare condition, despite the frequency of perforation associated with sigmoid diverticular disease. Anorectal involvement, although not often seen, manifests as draining fistulas and must be distinguished from Crohn's disease. ${ }^{1}$ Acute ulcerative diseases of the gastrointestinal tract and trauma also have been implicated as predisposing factors. Only rare bony extension or lymphatic spread 


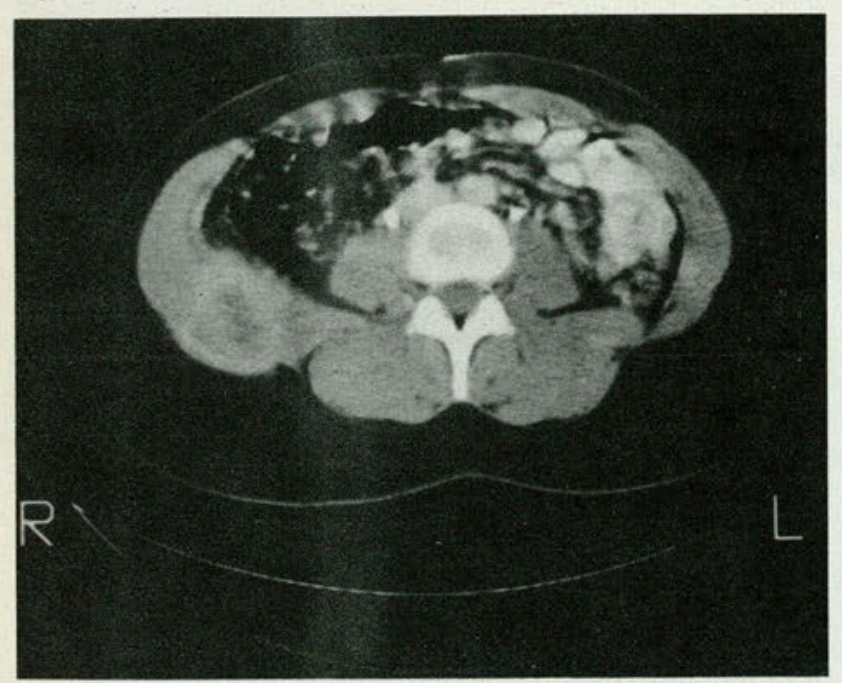

Figure 1. Computer tomogram illustrating retroperitoneal actinomycosis.

is seen. Involvement of the liver, lung, or kidney in the series by Putman and associates ${ }^{4}$ was by direct extension of the infectious process and not by hematogenous spread.

\section{Report of case}

A 33-year-old woman was seen in consultation regarding a mass in her right flank musculature. She noticed some soreness in this area following an aerobic exercise session. She had no related fever, chills, gastrointestinal, gynecologic, or genitourinary complaints. Her medical history was unremarkable, and she was well until 3 years prior, when acute appendicitis developed. At surgery, a ruptured appendix was removed. The area was drained widely, including the use of a drain that was brought out through a separate stab incision that was placed laterally in the flank. She had an uncomplicated recovery and was well until seen at this time.

Physical examination revealed a healthy-appearing woman with tenderness and a distinct fullness in the right flank. The findings of the remainder of the examination were unremarkable. Results of a complete blood cell count and urinalysis were normal. The erythrocyte sedimentation rate was 37 $\mathrm{mm} / \mathrm{h}$. Computer tomographic scanning of the abdomen revealed a 15-cm mass of low density, with a surrounding high-density capsule adjacent to the right quadratus lumborum and iliacus muscles (Fig 1). Diagnostic considerations included hematoma, abscess, or neoplasm.

The patient was taken to the operating room. A long oblique flank incision was made, and retroperitoneal dissection was carried out to the level of the quadratus lumborum muscle, where an abscess cavity was found. Approximately $70 \mathrm{~mL}$ of a pecu- liar thin, green-yellow pus containing many $2-\mathrm{mm}$ spherical aggregates was drained. The sidewalls of the cavity were aggressively debrided and sent for pathologic evaluation. The wound was packed open and drained.

Microscopic examination revealed the presence of Actinomyces organisms. Characteristic "sulfur granules" were seen. Higher-power magnification revealed mycelial filaments (Figs 2 and 3). Treatment with 5 million units of penicillin intravenously every 6 hours was begun. Anaerobic cultures yielded Bacteroides fragilis, and metronidazole was added to the treatment regimen. Actinomyces was identified on culture after 2 weeks.

The drains were removed gradually, and the wound healed without incident. The patient was discharged on the tenth day and continued treatment with penicillin VK (1 g orally four times daily) for 3 weeks. Results of a follow-up computer tomographic scan 6 months later showed no reaccumulation in the abscess cavity, and the patient has felt well since.

\section{Discussion}

The etiology of actinomycotic infections was subject to some debate in past years. Bostroem collected Actinomyces that grew under aerobic conditions at room temperature. He postulated that the organisms occurred freely in nature and had their natural habitat on grasses and grains (the exogenous theory). ${ }^{4} \mathrm{~A}$ high distribution of the disease among rural inhabitants and agricultural workers made this an attractive theory. ${ }^{5}$

However, in the 1950s the work of Wright, Lord, Trevett, Naesland, and other researchers carried out during the previous 40 years substantiated Israel and Wolf's beliefs. ${ }^{4}$ Specifically, the causative agent was a delicate, preferably anaerobic organism that grew only at body temperature and was not found outside the bodies of animals or human beings (the endogenous theory). ${ }^{4}$

Actinomycosis and nocardiosis are two distinct disease entities with grossly similar morphologic and clinical characteristics. Actinomyces are non-acid-fast organisms, and anaerobic (or somewhat microaerophilic). The Nocardia are acid-fast organisms to variable degrees, and grow aerobically. Actinomyces grow well only at incubator temperatures; Nocardia can 


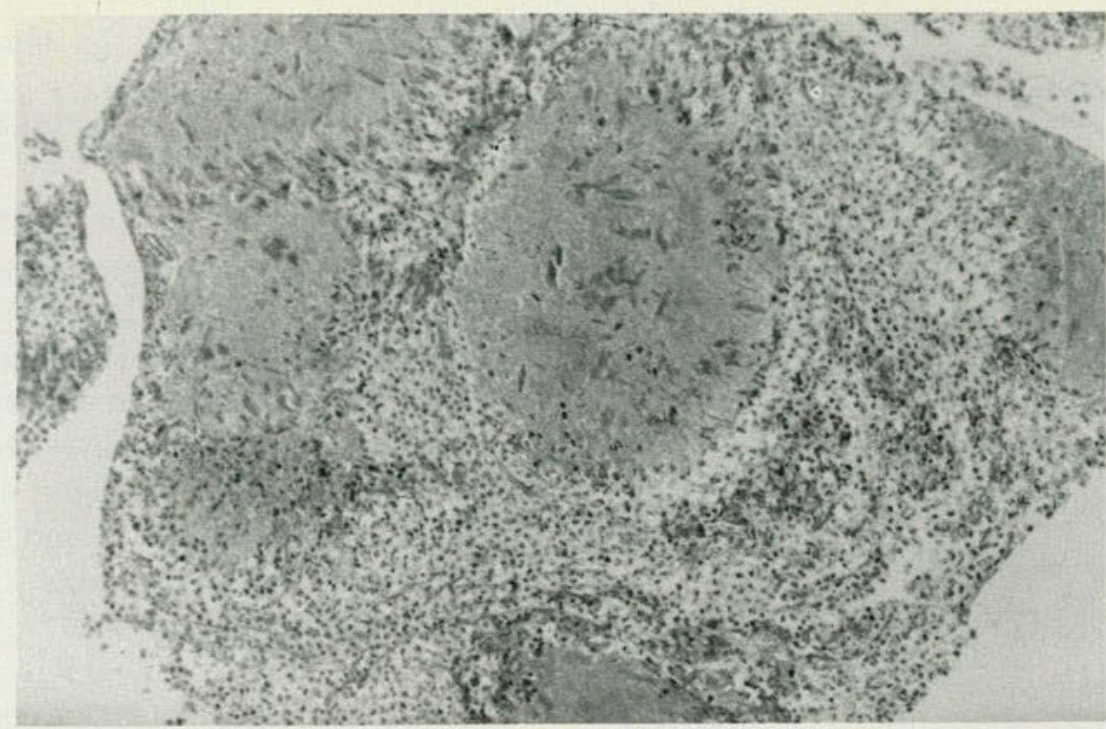

Figure 2. Actinomyces israelii (hematoxylin-eosin, original magnification $\times 200$ ).

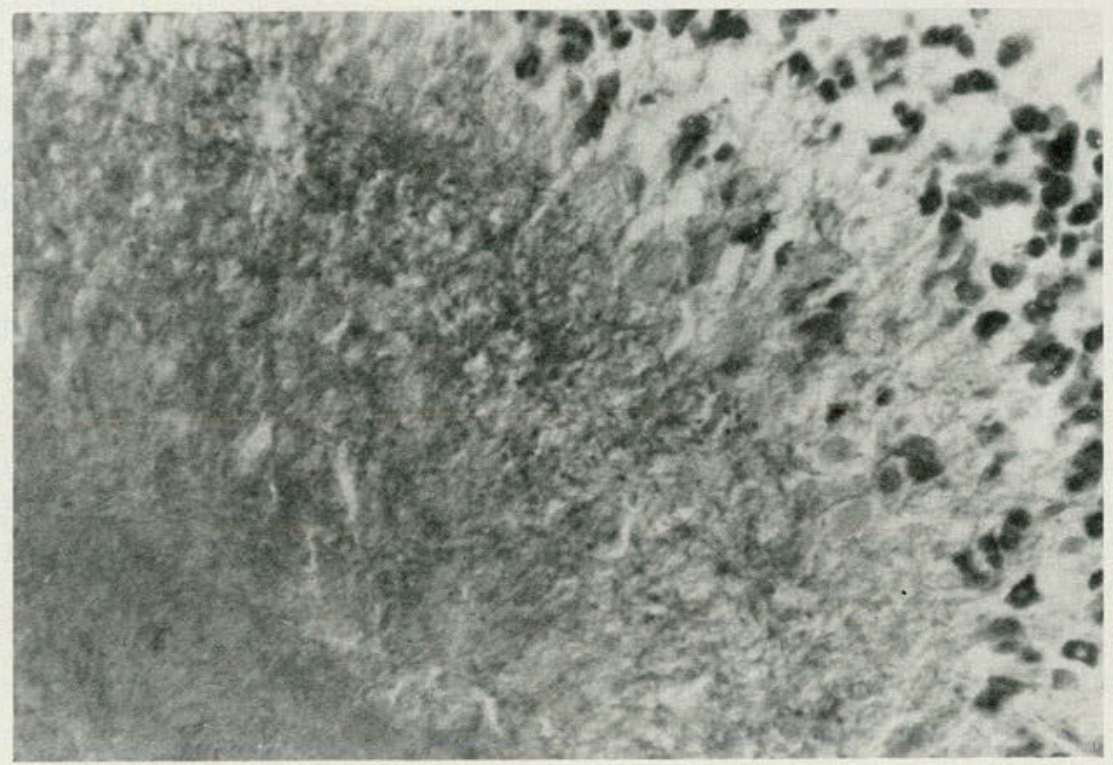

Figure 3. High-power magnification showing mycelial filaments and surrounding mononuclear inflammatory response (hematoxylin-eosin, original magnification $\times 1000$ ).

grow at room or incubator temperatures and require no special culture media.

The distinguishing criterion of the entire order Actinomycetales revolves around the propensity for filamentous growth with true branching. A collection of filaments is known as the mycelium (Table). ${ }^{2}$

\section{Pathologic aspects}

Considering the frequent occurrence of various gastrointestinal perforations and the rela- tive rarity of actinomycosis, one wonders why this infection is not more common. Why an organism exhibiting as little animal pathogenicity as $A$ israelii should be capable of assuming such virulence in humans is perplexing. Attempts at reproducing the disease by inoculating laboratory animals with pure cultures alone have seldom been successful. ${ }^{2}$ This may be due to previous sensitization to the organism, with improved host response. ${ }^{6}$

Host resistance and virulence of certain 


\begin{tabular}{|l|}
\hline \multicolumn{1}{c|}{ Table } \\
\multicolumn{1}{c|}{ Order Actinomycetales } \\
\hline $\begin{array}{l}\text { Mycelium rudimentary or absent } \\
\text { Mycobacteriaceae } \\
\text { Mycobacterium }\end{array}$ \\
True mycelium produced \\
Actinomycetaceae (fragment into bacillary and \\
coccoid elements) \\
Actinomyces \\
Anaerobic and non-acid fast \\
(Actinomyces israelii) \\
Nocardia \\
Aerobic \\
Non-acid fast \\
Acid fast \\
$\quad$ Nocardia asteroides) \\
Streptomycetaceae (do not fragment) \\
Streptomyces \\
Micromonospora \\
\hline Source: Adapted with permission from Peabody JW Jr, Seabury JH: \\
Actinomycosis and nocardiosis. J Chronic Dis 1957;5:374-403. \\
Copyright 1957, Pergamon Press, Inc.
\end{tabular}

strains, as well as stasis of the fecal stream, have all been offered as contributing factors. ${ }^{4}$ It is significant that certain bacteria are found in actinomycotic infections. Holm, ${ }^{7,8}$ and later Glahn ${ }^{9}$ suggested that coexisting bacterial infections were essential for the development of actinomycosis. A synergistic mechanism is thought to be operative.

On physical examination, the lesions are poorly circumscribed with overlying brawny, red skin. The lesions appear fixed and indurated. The lesions gradually soften over a period of weeks, and cutaneous sinuses appear. Areas of abscess formation and fibrosis develop. Drainage may be serosanguineous and thin or grossly purulent, with yellow or brown sulfur granules. A honeycomb multiloculated appearance is often seen on entering the cavity and on cross-section.

Microscopically, microabscesses with liquifactive rather than caseative necrosis are found. Fibrosis with granuloma formation occurs. Suppuration consisting of an abundance of leukocytes, occasional lymphocytes, monocytes, plasma cells, and histiocytes is seen. The organism is not frequently seen on tissue sections, but, when present, it is seen as a colony of sulfur granules in a pool of pus. ${ }^{4}$ This microscopic picture supports the belief that these infections come about by the synergistic activity of several organisms.

\section{Diagnosis}

Microscopic and culture identification are essential for the definitive diagnosis. Demonstration of the mycelial filaments and gram-positive staining are needed for morphologic identification, and cultures are needed from the sidewall of the abscess cavity for successful culture identification of these organisms. Even then, with special media and extended culture times (in excess of 2 weeks), growth may not be demonstrated. ${ }^{4}$

When approached with adequate forethought, distinguishing this condition from carcinoma, diverticular disease, or Crohn's disease can be done expediently. Because actinomycosis is a great imitator, its diagnosis could take weeks to 6 years. ${ }^{1}$ In the series of 7 cases described by Davies and Keddie, ${ }^{1}$ the average time from onset of symptoms to diagnosis was 18 months, ranging from 1 week to 6 years. The clinical history is invaluable in these patients, as the latent period is preceded by some abdominal catastrophe involving perforated gut. A draining sinus tract may develop. However, cultures may yield only bacterial infection with Escherichia coli and staphylococci, while biopsy specimens show nonspecific inflammation. ${ }^{1}$ Protean manifestations, including weight loss, pyrexia, and microcytic anemia are frequent features. Erythrocyte sedimentation rates usually are elevated, but extreme leukocytosis is rare.

\section{Treatment}

Management principles have changed little since 1960. At that time, Peabody and Seabury ${ }^{2}$ emphasized intensive and prolonged antibiotic therapy in addition to appropriate surgical drainage and curettage, with radical excision of sinus tracts as needed. ${ }^{10}$ When the condition is recognized early and treated appropriately, tetracyclines are equally as effective as penicillins. ${ }^{11}$ However, the drug of choice is intravenously administered penicillin (10 to 20 million U/d) for 4 to 6 weeks. This regimen is followed by orally administered penicillin ( 2 to $4 \mathrm{~g} / \mathrm{d}$, depending on patient toler- 
ance) for an additional 6 to 12 months. Varkey and colleagues ${ }^{12}$ have advocated this regimen for deep-seated infection. Dosing schedules can not be standardized, but must be based on clinical response. ${ }^{10}$

The value of erythromycin and lincomycin has been reported. Lincomycin is particularly advantageous in the face of bony involvement. ${ }^{10}$ Other modalities include hyperbaric oxygen used in conjunction with penicillin. Manheim and coauthors ${ }^{13}$ described this treatment in a case of resistant perirectal actinomycosis when involvement of adjacent structures precluded adequate surgical extirpation.

\section{Conclusion}

Actinomycosis is an uncommon, but by no means rare, disease. The historical and pathologic aspects of this disease have been reviewed, along with guidelines for accurate diagnosis and treatment. As always, logical review of the patient's history, along with the appropriate testing will lead to the proper diagnosis. In this case, proper diagnosis is necessary to avoid morbidity and delay in therapy for a problem that can be readily treated.
We acknowledge the assistance of W.L.Hoppes, MD, Massillion, Ohio.

1. Davies M, Keddie NC: Abdominal actinomycosis. Br J Surg 1973;60:18-22

2. Peabody JW, Jr, Seabury JH: Actinomycosis and nocardiosis. J Chronic Dis 1957;5:374-403.

3. Cope VZ: Actinomycosis. New York, Oxford University Press, 1938.

4. Putman HC, Dockerty MD, Waugh JM: Abdominal actinomycosis. Surgery 1950;28:781-800.

5. Porter IA: Actinomycosis in Scotland. Br Med J 1953;10841086.

6. Mathieson DR, Harrison R, Hammond C, et al: Allergic reaction of the Actinomycetes. Am J Hygiene 1935;21:405-421.

7. Holm P: Studies on the etiology of human actinomycosis: I, The other microbes of actinomycosis and their importance. Acta Pathol Microbiol Scand 1950;27:736-751.

8. Holm P: Studies on the etiology of human actinomycosis: II, Do the other microbes possess virulence? Acta Pathol Microbiol Scand 1951;28:391-406.

9. Glahn M: The pathogenesis of cervicofacial actinomycosis. Acta Chir Scand 1954;108:193-204.

10. Eastridge CE, Prather R, Hughes FA, Jr, et al: Actinomycosis: A 24-year experience. South Med J 1972;65:839-843.

11. Mandell GL, Douglas RG, Bennett JE: Principles and Practice of Infectious Disease, ed 3. New York, Churchill Livingstone Inc, 1990, p 1940.

12. Varkey B, Landis FB, Tang TT, et al: Thoracic actinomycosis. Arch Intern Med 1974;134:689-693.

13. Manheim SD, Voleti C, Ludwig A, et al: Hyperbaric oxygen in the treatment of actinomycosis. JAMA 1969;210:552-553. 